\title{
Thermodynamic Properties of the Mars Atmosphere Evaluated by Saha and Thomas-Fermi Models
}

\author{
Vyacheslav V. Shumaev ${ }^{1}$, Victor V. Kuzenov ${ }^{1,2}$
}

${ }^{1}$ Department of Thermal Physics, Bauman Moscow State Technical University, Moscow, 105005, Russian Federation

${ }^{2}$ A.Yu. Ishlinsky Institute for Problems in Mechanics RAS, Moscow, 119526, Russian Federation

Keywords: ionization equilibrium model, Saha model, Thomas-Fermi model, thermodynamic properties, mixture of elements, atmosphere of Mars.

\begin{abstract}
In this work the thermodynamic properties (the full pressure $\mathrm{P}$, the internal energy per unit mass $\mathrm{E}$, the average ionization degree $\alpha$ ) of mixture of gases, evaluated by the Thomas-Fermi model and ionization equilibrium (Saha) model, are compared. It was used the mixture of oxygen, carbon and nitrogen in the proportion of the Mars atmosphere. The range of state parameters: the average density $\rho=10-3 \div 10-7 \mathrm{~g} / \mathrm{cm} 3$, the temperature $\mathrm{T}=(1 \div 100) \mathrm{eV}$.
\end{abstract}

\section{Introduction}

For the some problems of science and technology, in particular for the atmospheric entries of spacecraft calculations, there is a need in data on the properties of substances at temperatures about thousands of degrees Kelvin and above [1-5]. This data can be obtained not only by the ionization equilibrium (Saha) model [1, 5], but the finite temperature Thomas-Fermi model (TF model) [1, 2, 6-9], because the region of joint applicability of these models may include this range of state parameters: average density $\rho=10^{-3} \div 10^{-7} \mathrm{~g} / \mathrm{cm}^{3}$, the temperature $T=(1 \div 100) \mathrm{eV}$ [5-9].

In this paper the region of quantitative proximity of these models, formulated for the mixture of substances is discussed. The results of thermodynamic properties evaluation are compared for the mixture of oxygen, carbon and nitrogen in the proportion of the Mars atmosphere. Thermodynamic properties are the full pressure $P$, the internal energy per unit mass $E$, the average ionization degree $\alpha$. The data by the TF model is obtained by authors $[8,9]$, the data by the Saha model is taken from the work [5]. Note that the substance under these temperatures $T$ and densities $\rho$ satisfy the conditions of the local thermodynamic equilibrium $[1,2,5]$.

\section{The Finite Temperature Thomas-Fermi Model for the Mixture of Elements}

The mixture of $N$ components at the given temperature $\theta=k_{\mathrm{B}} \cdot T$ ( $k_{\mathrm{B}}$ is the Boltzmann constant) and the average density $\rho$ is considered. This mixture consists of the sum of spherical atomic cells with radius $r_{0 i}$, which have nuclei (obey the Boltzmann statistics) and electrons (obey the Fermi-Dirac statistics). Note, that in the local thermodynamic equilibrium conditions chemical potentials $\mu_{\mathrm{i}}$ must be equal.

So, the evaluation of the TF potential for the different cells for the mixture are brought to the system of nonlinear differential equations of 2-nd order with appropriate boundary conditions:

$$
\begin{aligned}
& \frac{d^{2}}{d x^{2}} \phi_{i}(x)=a_{i} x I_{1 / 2}\left(\frac{\phi_{i}}{x}\right), \quad(i=1,2, \ldots N), \\
& \phi_{i}(0)=\frac{Z_{i}}{\theta r_{0 i}}, \phi_{i}(1)=\left.\frac{d \phi_{i}}{d x}\right|_{x=1}=\frac{\mu_{i}}{\theta}=-\eta_{i},
\end{aligned}
$$

where $x=r / r_{0 i}, \phi_{i}(x) / x=\left(V_{i}(r)+\mu_{i}\right) / \theta, a_{i}=4(\sqrt{2 \theta} / \pi) r_{0 i}^{2}, r_{0 i}=1,388\left(A_{i} / \rho_{i}\right)^{1 / 3}, \quad I_{1 / 2}(x)=\int_{0}^{\infty} \frac{y^{\frac{1}{2}} d y}{1+\exp (y-x)}$ is the Fermi-Dirac function, $y=p^{2} / \theta, A_{i}, Z_{i}$ is atomic weight and charge number of the component 
with index $i$. During the solution of the system (1)-(2) radii $r_{0 i}$ and partial densities $\rho_{i}$ are selected in a way to satisfy these conditions:

$$
\frac{1}{\rho} \sum_{i=1}^{N} m_{i}=\sum_{i=1}^{N} \frac{m_{i}}{\rho_{i}}, \eta_{i}=\eta_{j}=\eta=-\frac{\mu}{\theta}, \quad \forall i, j .
$$

The procedure of evaluating the degree of ionization, the pressure and the relative internal energy for the case of a single substance is described in $[2,7,8]$. In the case of the mixture the Thomas-Fermi mixing of elements method is used [10]: the contribution of ions is handled as a single species with mean atomic number $\bar{Z}=\sum_{i} x_{i} \cdot Z_{i}$ and weight $\bar{A}=\sum_{i} x_{i} \cdot A_{i}, x_{i}, Z_{i}, A_{i}$ are the number fraction, the charge and atomic weight of $i$-ого of the component with index $i$ respectively. For the electronic contribution of the mixture the densities of all species are adjusted in order to fulfill conditions (1). Electronic contribution to thermodynamic functions of the mixture is evaluated by summing up the values for each element, using the algorithms for the single substance (partial densities $\rho_{i}$ are used here), and adding the mass fraction coefficient $m_{i}=x_{i} \cdot A_{i} / \bar{A}$, to each of this value.

\section{The Calculation Results. The comparison with the Ionization Equilibrium (Saha) Model}

The test evaluation was made for the mixture of 10 elements with charge numbers $Z=10,20, \ldots$, 100 and $m_{i}=A_{i}$. The chemical potential $\eta=-\mu / \theta=-\phi_{\mathrm{i}}(1)$ of the mixture at $\rho=1 \mathrm{~g} / \mathrm{cm}^{3}$, obtained by authors, is compared with the known data and presented in the Table.

Table 1: The comparison of calculation results with the known data

\begin{tabular}{llll}
\hline \multicolumn{1}{c}{$T,[\mathrm{keV}]$} & \multicolumn{1}{c}{$\eta$ from the present work } & \multicolumn{1}{c}{$\eta$ from [2] } & error $\Delta, \%$ \\
\hline 10 & 10.11783 & 10.11881 & 0.01 \\
1 & 6.91901 & 6.92075 & 0.03 \\
0.1 & 4.54926 & 4.55316 & 0.09 \\
0.01 & 2.48412 & 2.49378 & 0.40
\end{tabular}

It is observed the agreement of $\eta$ with an error up to $0.4 \%$ and the error decreases then the temperature $T$ raises.

The Fig. 1 represents the average ionization degree $\alpha$, the full pressure $P$ (the pressure of electrons and ions), the internal energy per unit mass $\mathrm{E}$ depending on $T$. These functions are evaluated by the TF model, and are taken from the literature data, that uses the ionization equilibrium (Saha) model [5]. It was used the mixture of oxygen, carbon and nitrogen in the proportion of the Mars atmosphere. Partial composition of the Mars atmosphere (by chemical elements): $32.6 \% C ; 2.1 \% N ; 65.3 \% O$ [5]. Note that only the proportion between ion sorts with different ionization degrees is temperature dependent. The thermodynamic properties are compared in the area, where both models work: $T=1 \div 100 \mathrm{eV}, \rho=10^{-7} \div 10^{-3} \mathrm{~g} / \mathrm{cm}^{3}$ [5-9].

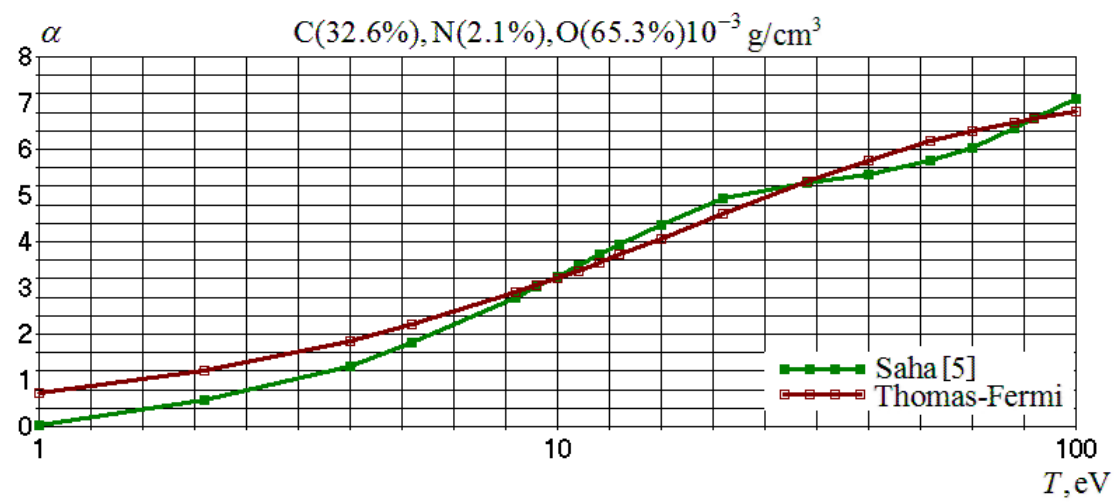



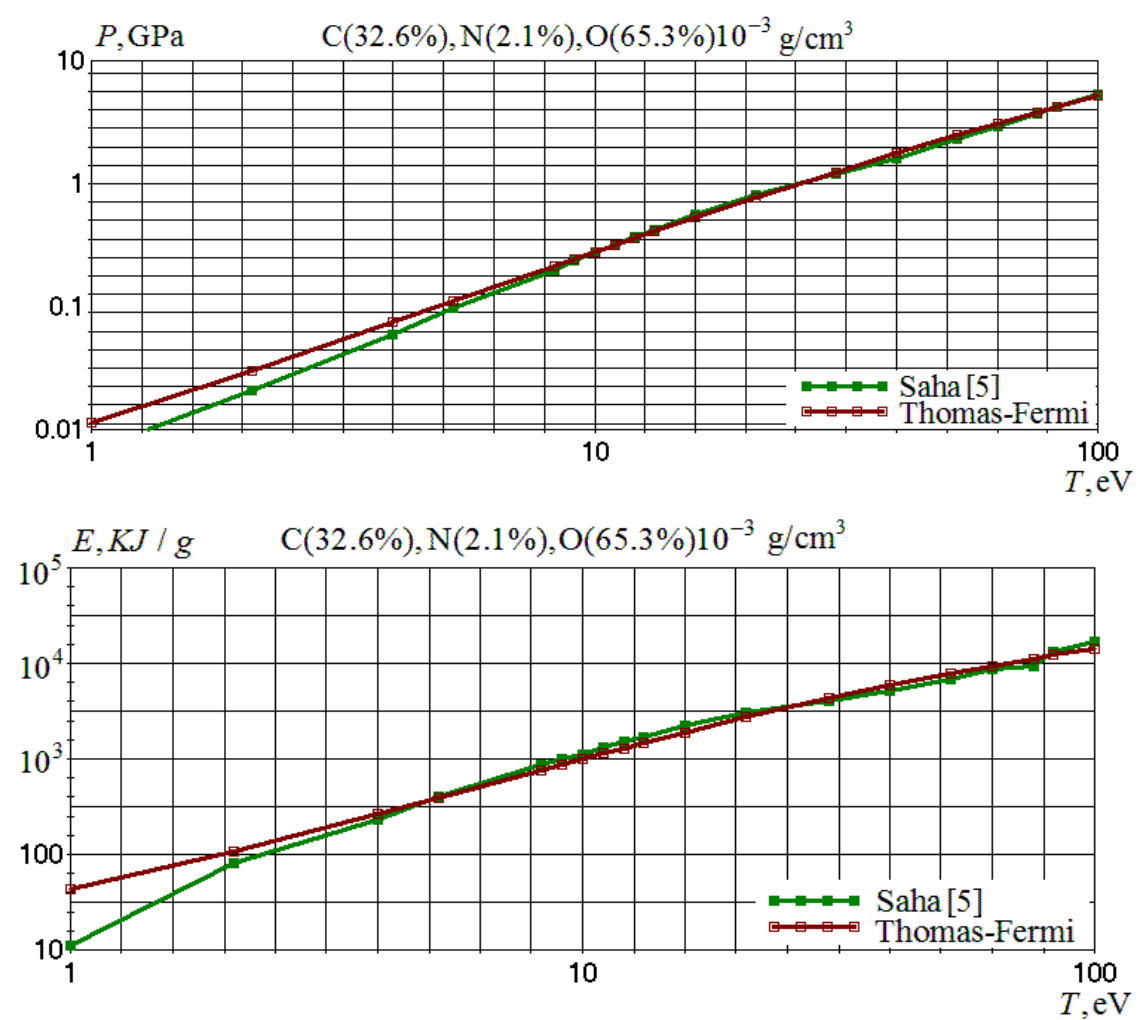

Fig. 1: The degree of ionization $\alpha$, the full pressure $P$ and the relative internal energy $E$ depending on the temperature $T$, evaluated by the Saha model [5] and the finite temperature Thomas-Fermi model for the mixture of carbon, oxygen and nitrogen at the average density $10^{-3}$ $\mathrm{g} / \mathrm{cm}^{3}$.

The Fig. 2 represents the relative difference $\Delta$ between the Saha model results [5] and the finite temperature TF model results depending on the temperature $T$. The relative difference $\Delta$ is:

$$
\Delta=\frac{\left|P_{T F}-P_{\text {Saha }}\right|}{\min \left(P_{T F}, P_{\text {Saha }}\right)}, \%
$$

where $P_{T F}, P_{\text {Saha }}$ is the one of the thermodynamic properties $(P, E, \bar{Z})$, evaluated by the TF model and the Saha model respectively.

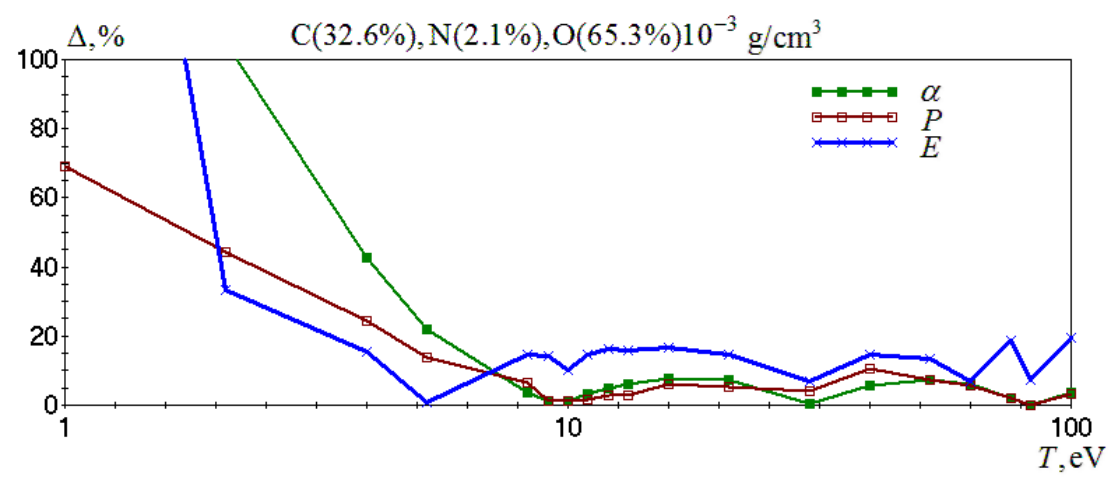




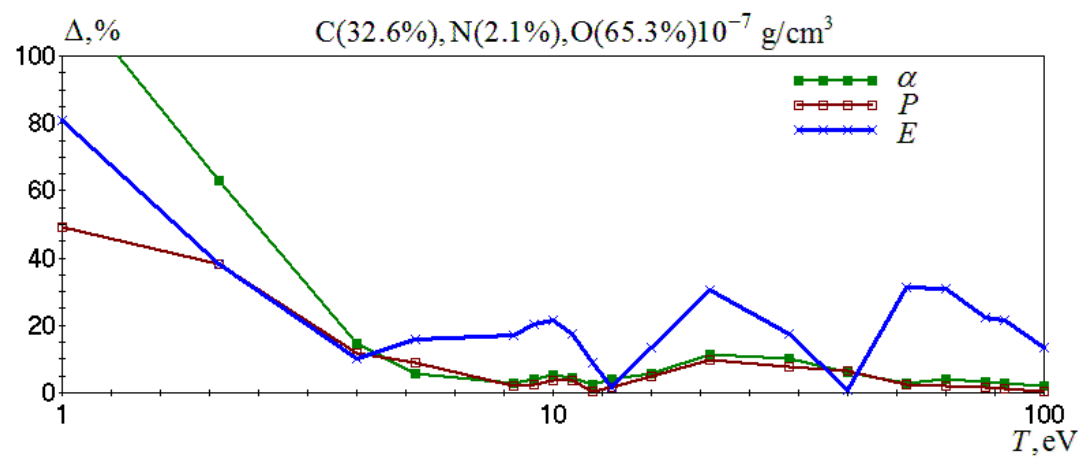

Fig. 2: The relative difference $\Delta$ between the Saha model results [5] and the finite temperature Thomas-Fermi model results depending on the temperature $T$ for the mixture of carbon, oxygen and nitrogen at the average density $10^{-3}$ and $10^{-7} \mathrm{~g} / \mathrm{cm}^{3}$.

The Fig. 2 shows, that in the temperature range $(1 \div 6) \mathrm{eV}$ the difference between the Saha model results and the TF model results is intolerably huge. This happens, because the thermodynamic properties, obtained by the TF model, are inaccurate in this temperature range [6-9]. Otherwise, at the temperature $T \geq 10 \mathrm{eV}$ both models show similar results: the relative difference $\Delta$ is approximately $10 \%$ for the pressure $P$ and the degree of ionization $\alpha$ and approximately $20 \%$ for the relative internal energy $E$ and doesn't decrease when the temperature $T$ rises.

\section{Conclusions}

The area of quantitative proximity for the Saha model and the finite temperature Thomas-Fermi model was searched for the mixture of oxygen, carbon and nitrogen in the proportion of the Mars atmosphere. On this purpose for the plasma of these elements (for the region of state parameters $T=$ $10 \div 100 \mathrm{eV}$ and $\rho=10^{-7} \div 10^{-3} \mathrm{~g} / \mathrm{cm}^{3}$ ) the degree of ionization $\alpha$, the full pressure $P$ and the relative internal energy $E$ depending on the temperature $T$ were compared. It was obtained, that at the temperature $T \geq 10 \mathrm{eV}$ both models show similar results, but at lower temperatures the discrepancy takes place. It can be explained as poor applicability of Thomas-Fermi model at low temperatures.

\section{Acknowledgements}

The research work was supported by the Ministry of Education and Science of the Russian Federation (Project No. 13.79.2014/K).

\section{References}

[1] Zel'dovich Ya.B. \& Raizer Yu.P. Physics of Shock Waves and High-Temperature Hydrodynamic Phenomena. New York: Dover Publications, 2002.

[2] Nikiforov A.F., Novikov V.G. \& Uvarov V.B. Quantum-Statistical Models of Hot Dense Matter. Methods for Computation Opacity and Equation of State. Basel: Birkhauser Verlag, 2005.

[3] Kuzenov V.V. \& Ryzhkov S.V. Numerical Modeling of Magnetized Plasma Compressed by the Laser Beams and Plasma Jets. Problems of Atomic Science and Technology, 83, pp. 12-14, 2013.

[4] Ryzhkov S.V. Current state, problems, and prospects of thermonuclear facilities based on the magneto-inertial confinement of hot plasma. Bulletin of the Russian Academy of Sciences. Physics, 78, pp. 456-461, 2014.

[5] Boyko Yu. V., Grishin Yu. M., Kamrukov A. S. et al.: Thermodynamic and Optical Properties of Ionized Gases at Temperatures to $100 \mathrm{eV}$. Boca Raton: CRC Press, 1991.

[6] Kirzhnits D.A. The limits of applicability of the quantum-classical Equation of State of matter. JETP, 8, pp. 1081-1090, 1959 
[7] Dyachkov S. \& Levashov P. Region of validity of the finite-temperature Thomas-Fermi model with respect to quantum and exchange corrections. Phys. Plasmas, 21, 052702, 2014.

[8] Kuzenov V.V., Ryzhkov S.V. \& Shumaev V.V. Thermodynamic properties of magnetized plasma evaluated by Thomas-Fermi model, Applied Physics, 3, pp. 22-25, 2014.

[9] Kuzenov V.V. \& Shumaev V.V. Description of the thermodynamic properties of plasma in Saha and Thomas-Fermi models, Applied Physics, 2, pp. 32-36, 2015.

[10] More R. M., Warren K., Young D., and Zimmerman G. A new quotidien equation of state (QEOS) for hot dense matter. Phys. Fluids, 31, pp. 3059-3078, 1988. 